\title{
Cosmic Illusions
}

\author{
Bernard H. Lavenda \\ Università degli Studi, Camerino, Italy \\ Email: info@bernardhlavenda.com
}

Received April 17, 2013; revised May 21, 2013; accepted June 23, 2013

Copyright (C) 2013 Bernard H. Lavenda. This is an open access article distributed under the Creative Commons Attribution License, which permits unrestricted use, distribution, and reproduction in any medium, provided the original work is properly cited.

\begin{abstract}
A critique of black-hole-black-body radiation, black-hole thermodynamics, entropy bounds, inflation cosmology, and the lack of gravitational aberration is presented. With the exception of the last topic, the common thread is the misuse of entropy and, consequently, the second law. Hawking's derivation of the entropy loss due to black hole emission rests on Kirchhoff's radiation law which equates the rates of absorption and emission of energy in any given frequency interval. Black-body radiation cannot, therefore, be used as a mechanism for black-hole evaporation. A derivation of the Planck factor from an exponential Doppler shift shows why the temperature cannot be proportional to the acceleration; accelerations do not cause Doppler shifts. Inflationary cosmology is based on a misconception that the adiabatic condition of Einstein's equations hold, and, yet, there can be an enormous increase in the entropy. The cause for the increase is a negative pressure which contradicts the thermodynamic definition of positive pressure as the derivative of the entropy with respect to the volume times the temperature: Increases in volume cause corresponding increases in the entropy. A first-order phase transition cannot occur under adiabatic conditions, cannot generate entropy, and the latent heat cannot be used to reheat the universe. Finally, a negative pressure is invoked to explain the absence of gravitational aberration, assuming that gravity propagates at the speed of light.

It is the only physical theory of universal content which I am convinced will never be overthrown, within the framwork of applicability of its basic concepts.
\end{abstract}

Albert Einstein on Thermodynamics

Keywords: Black-Hole Radiation; Black-Hole Thermodynamics; Entropy Bounds; Inflationary Cosmology; Gravitational Aberration

\section{Introduction and Summary}

The motto that history always repeats itself is not always true. The comparison of Planck's derivation of the blackbody spectrum and its associated entropy to the derivation of Hawking radiation and Bekenstein entropy of a black-hole turns up vast differences. First, and foremost, Planck always had an experimental verification of his formulas. Planck's assumption of the Wien distribution was contested by the results of Ruben and Kurlbaum in the long wavelength region, and led him to the search of a new distribution that would make the spectral density proportional to the absolute temperature in that region [1]. Moreover, the fitting parameters of that distribution led to the discovery of not one, but two universal constants, which along with $c$, the velocity of light, and $G$, the gravitational constant, would be "independent of particular bodies or substances", and would "necessarily retain their significance for all times and for all cultures, including extraterrestial and non-human ones". Planck re- ferred to them as "natural units", and would "retain their natural significance as long as the laws of gravitation and the propagation of light in vacuum, and the two laws of thermodynamics retain their validity" [2].

If Planck had thought through the natural units he was considering he would have realized that they constituted extremely high energies of $1.22 \times 10^{19} \mathrm{Gev}$, extremely short times of $5.4 \times 10^{-44} \mathrm{sec}$, and extremely short lengths of $1.6 \times 10^{-35} \mathrm{~m}$. It was only after the general acceptance of the big bang that such a Planck scale could have been realized immediately after the bang. On such a Planck scale, $G$ would be comparable to the other forces of nature. Since there is no known theory that can probe this scale, reliance must be made on the rest of what Planck considered immutable, namely the two laws of thermodynamics.

Hawking radiation is thermal radiation that is predicted to be spontaneously emitted by black-holes. It is a consequence of the steady conversion of quantum vac- 
uum fluctuations into pairs of particles, one of which is ejected at the event horizon and escapes to infinity, while the other is trapped within the event horizon. Since this radiation reduces the mass of a black-hole, Hawking radiation is said to be responsible for black-hole evaporation.

A black-hole is thought to be the collapse of a star in which its matter and energy is veiled to an external observer behind an event horizon. Thus, a thermodynamic description from an observer's view cannot be based on the mass and radiation before the black-hole was formed because they are no longer observable. Associating an entropy with a black-hole provides a handle on the thermodynamics. The question comes to mind: a thermodynamics of what? Assuming there are many scenarios of black-hole formation, the microscopic interpretation of entropy as a measure of the number of complexions that are all compatible with a single macro-state would quantify the multiple ways in which a black-hole could be formed. Once a black-hole is formed, information seems to have disappeared. Since entropy is a measure of missing information, this would provide yet another reason of associating entropy with a black-hole.

The second law guarantees that the entropy of an isolated system will tend to increase until it reaches a maximum. But how large is this maximum? Not wanting to appeal to statistical mechanical models, it should be possible to argue from thermodynamic principles how large this maximum should be. Because maximum entropy measures information, it should provide a bound on information capacity.

Entropy has also been implicated in resolving the seemingly paradoxes associated with the standard model of cosmology which refers to an adiabatically expanding, radiation-dominated universe that is supposedly welldescribed by a Robertson-Walker metric. The paradoxes are due to 1) the horizon problem whereby causally disconnected regions could evolve into a homogeneous universe; and 2) the flatness problem whereby the only universe that could reach a critical energy in the present epoch would be a flat universe since a closed universe would have achieve it in Planck's time while an open universe would see its energy density rapidly dwindle away. According to the inflationary scenario, if the adiabatic assumption would be dropped in the standard model, these paradoxes would disapper.

In all these cases there is the common thread of using thermodynamic laws in the absence of experimental verification. More specifically, it is the second law and entropy which hold center stage. But, the "entropy" used in the first two cases has no relation to the second law, and, hence, is not an entropy, and relinquishing the constancy of the entropy while maintaining Einstein's adiabatic equations is a contradiction. It is the purpose of this pa- per to redress these issues.

A simplified derivation of the black-body spectrum for black-hole radiation and the Unruh temperature is marred on, at least, two accounts: 1) acceleration does not cause a Doppler shift; and 2) the integral of the spectral density function over all frequencies diverges. Hence, absolute temperature cannot be proportional to acceleration, in general, and surface gravity, in particular. The expression for the entropy of a black-hole violates both the second and third laws of thermodynamics, and there is no bound on the entropy-to-energy ratio, precisely because it is a decreasing function of the energy: The entropy-to-energy ratio can never be less than to the derivative of the entropy with respect to energy. Black-body radiation cannot be used as a mechanism of black-hole evaporation precisely because it obeys Kirrchhoff's radiation law asserting that radiation cannot upset a state of thermal equilibrium [1, p. 64].

Inflation cosmology violates the adiabatic nature of Einstein's equations. Since the Robertson-Walker metric does not predict that the ratio of a circle to its radius will be greater (less) than $2 \pi$ for geometries of constant negative (positive) curvature, it cannot describe non-Euclidean geometries of constant curvature. Thermodynamically derived relations for the energy and pressure are compared with those obtained from Einstein's equations. For space-time with constant Gaussian curvature, Einstein's equations require the pressure to be negative [3]. In the case of vanishing pressure, they reduce to the virial theorem, and Newton's law. Pressure has the effect of causing deceleration which causes a compression of the cosmological fluid, which, in turn, increases the pressure, in violation of Le Châtelier's principle. In general, negaive pressures, invoked in expansion, must lead to a decrease in volume if entropy is to increase so that the enormous increase in entropy predicted by inflation cannot occur. The latent heat of a phase transition cannot be used to re-heat a universe, and a first-order phase transition does not generate entropy.

Finally, the condition for the absence of gravitational aberration leads to a negative pressure of exactly the same magnitude, but of opposite sign, as the relativistic equations of state of degenerate stars. These equations show that ratio of the pressure to rest energy density ratio is always proportional to the ratio of the Schwarzschild radius to the radius of the star.

\section{Do Black Holes Emit Black-Body Radiation?}

Much of modern cosmology is based upon an inexorable chain of analogies [4]. A case in point is the conclusion that black holes emit black-body radiation, which implies that the temperature be proportional to surface gravity. 
A simplified derivation is based on one-dimensional hyperbolic motion that was derived by Born in 1909, and a year latter by Sommerfeld. It can be found in almost any text on relativity [5]. For a particle moving under uniform gravitational acceleration,

$$
a=\frac{\mathrm{d}}{\mathrm{d} t}\left(\frac{v}{\sqrt{1-v^{2} / c^{2}}}\right)
$$

will be constant so that integration, with initial conditions $v(0)=0$ at $t=0$, simply gives

$$
a t=\frac{v}{\sqrt{1-v^{2} / c^{2}}}=c \sinh (\bar{v} / c),
$$

where $\bar{v}$ is the hyperbolic measure of the velocity. Rearranging results in

$$
v=\frac{\mathrm{d} x}{\mathrm{~d} t}=\frac{a t}{\sqrt{1+(a t)^{2} / c^{2}}}=c \tanh \bar{v} / c .
$$

Inverting (3) gives

$$
\bar{v}=c \tanh ^{-1}(v / c)=\frac{c}{2} \ln \left(\frac{1+v / c}{1-v / c}\right) .
$$

An expression for $\bar{v}$ can be obtained from what appears as time-dilation,

$$
d \tau=\sqrt{1-\frac{v^{2}}{c^{2}}} d t
$$

Consider two coordinate systems, $K^{\prime}$, which is moving at a velocity $v$ with respect to system $K$. We know that the two systems will be related by the Lorentz transformation. If we consider the motion at the origin of the $K^{\prime}$, its time $\tau$ will be related to the time $t$ of the $K$ system by

$$
\tau=\sqrt{1-\frac{v^{2}}{c^{2}}} t
$$

which expresses the well-known effect of time-dilation: Clocks in motion appear to run slower. Sometimes, it is expressed in its infinitesimal form, (5), but, because the velocity is uniform it integrates simply to (6). Not so in the case of uniform acceleration!

Since the velocity is not uniform, we must introduce (3) into (6); integration then gives

$$
\tau=\int_{0}^{t} \frac{\mathrm{d} s}{\sqrt{1+(a s)^{2} / c^{2}}}=\frac{c}{a} \sinh ^{-1}(a t / c),
$$

which, upon inverting, results in

$$
a t=\sinh (a \tau / c) \text {. }
$$

This is not time-dilation given by (6). In other words, frequency is to be associated with inverse time, not the inverse infinitesimal increment in time. Moreover, a comparison of (2) and (7) leads to the identification $\bar{v}=a \tau$, and once this identification is made, there results

$$
\mathrm{e}^{a \tau / c}=\sqrt{\frac{1+v / c}{1-v / c}}
$$

from (4). This is supposedly is an exponential Doppler shift caused by uniform acceleration. Nothing could be further from the truth!

Consider a light signal that is sent out at time $t_{o}$, reflected at time $t_{r}$, and returns in time $t_{b}$. The laboratory time, $t$, is the arithmetic average,

$$
t=\frac{1}{2}\left(t_{o}+t_{b}\right)
$$

while the distance covered is proportional to half of the time difference,

$$
r / c=\frac{1}{2}\left(t_{b}-t_{o}\right) .
$$

The uniform velocity is the ratio of (10) to (9), $v=r / t$. Taking the sum and difference of (9) and (10), in turn, give

$$
t_{b}=(1+v / c) t, \quad \text { and } \quad t_{o}=(1-v / c) t .
$$

Taking the product of the two expressions, and then the positive square root of the two relations give

$$
\sqrt{t_{o} t_{b}}=\sqrt{1-v^{2} / c^{2}} t
$$

Comparing (12) with (6), we conclude that the proper time is the geometric mean time,

$$
\tau=\sqrt{t_{o} t_{b}}
$$

which can never be superior to the laboratory time, (9), because of the arithmetric-geometric inequality. This is mathematical explanation of time-dilation.

Now, divide the former time by the latter in (11) to get

$$
\frac{t_{b}}{t_{o}}=\frac{1+v / c}{1-v / c} .
$$

Take the square root of both sides and then their logarithms to give

$$
\frac{c}{2} \ln \frac{t_{b}}{t_{o}}=\frac{c}{2} \ln \left(\frac{1+v / c}{1-v / c}\right) .
$$

On the strength of (4) and (8), (14) would necessarily imply

$$
a \tau=a \sqrt{t_{o} t_{b}}=\frac{c}{2} \ln \frac{t_{b}}{t_{o}},
$$

which is evidently wrong. The Doppler effect requires a uniform relative velocity between source and observer, not one given by (3). 
In fact, (6) is a second-order Doppler shift discovered by Ives and Stilwell back in 1938. Radiation traveling at an angle $\theta$ to the direction of a moving source has an observed frequency

$$
v^{\prime}=v \frac{\sqrt{1-v^{2} / c^{2}}}{1-(v / c) \cos \theta}
$$

The angle $\theta$, measured in the direction of the observer, was set equal to $\pi / 2$, and the conclusion of Ives and Stilwell was that a moving clock runs slower than a stationary one.

This is not to say that uniform acceleration, as is conventionally assumed, does not have an effect upon the rate of a clock relative to a clock in an inertial system. Instead of the reflection time being given by geometric mean, (13), in the presence of uniform acceleration, it will be given by harmonic mean $\left[\frac{1}{2}\left(1 / t_{o}+1 / t_{b}\right)\right]^{-1}[5, \mathrm{p}$. 403]. Thus, clocks will run even slower in a uniformly accelerating frame than in an inertial frame, as a consequence of the geometric-harmonic mean inequality. However, this has nothing to do with the Doppler effect which involves uniform velocities since time-dilation, (6), appears as a second-order Doppler effect. And if this no longer holds, there will no longer be a Doppler shift in general. Therefore, (8) is not an exponental Doppler shift caused by acceleration. However, let this not deter us from continuing the derivation of the spectral density of black-body radiation involving (8).

On the strength of (8), the exponential Doppler shift between emitted $v$ and observed frequency $v^{\prime}$ would be

$$
v^{\prime}=v \mathrm{e}^{a \tau / c} .
$$

For small times, (16) would reduce to

$$
v^{\prime} \approx v(1+a \tau / c)
$$

giving the wrong impression that the shift in frequency is due to acceleration, and not velocity.

To draw an illusionary analogy with black-body radiation, one [6] considers the frequency spectrum

$$
\left|\int_{-\infty}^{\infty} \mathrm{d} \tau \mathrm{e}^{i \omega \tau / 2 \pi} \mathrm{e}^{(i v c / a) \mathrm{e}^{a \tau / c}}\right|^{2}
$$

This is a double exponential integral which can be obtained in closed form. To this end, set $y=\mathrm{e}^{a \tau / c}$, and get

$$
\begin{aligned}
\int_{-\infty}^{\infty} \mathrm{d} \tau \mathrm{e}^{i \omega \tau / 2 \pi} \mathrm{e}^{i(\nu c / a) \mathrm{e}^{a \tau / c}} & =\frac{c}{a} \int_{-\infty}^{\infty} \mathrm{d} y y^{(i \omega c / 2 \pi a-1)} \mathrm{e}^{i(\nu c / a) y} \\
& =\frac{c}{a} \Gamma\left(\frac{i \omega c}{2 \pi a}\right)\left(\frac{i \nu c}{a}\right)^{-\omega c / 2 \pi a} \mathrm{e}^{\omega c / 4 a} .
\end{aligned}
$$

The relation for Gamma functions,

$$
\left|\Gamma\left(\frac{i \omega c}{2 \pi a}\right)\right|^{2}=\frac{\pi}{\frac{\omega c}{2 \pi a} \sinh \frac{\omega c}{2 a}},
$$

gives the spectral density as

$$
\left|\int_{-\infty}^{\infty} \mathrm{d} \tau \mathrm{e}^{i \omega \tau / 2 \pi} \mathrm{e}^{(i v c / a) \mathrm{e}^{a \tau / c}}\right|^{2}=\frac{c}{\omega a} \frac{1}{\mathrm{e}^{\omega c / a}-1} .
$$

Apart from the minor point that an integral over all frequencies of (18) diverges ${ }^{1}$, one considers $1 /\left(\mathrm{e}^{\omega c / a}-1\right)$ to be a bona fide Planck factor, and this necessitates defining the absolute temperature as [8]

$$
T=\frac{a \hbar}{k c} \text {. }
$$

Expression (19) is the well-known, and generally accepted, Unruh temperature, for which an accelerating thermometer would measure black-body radiation, whereas a stationary thermometer would measure no temperature at all!

The temperature, (19), is also the Hawking temperature [9] when we identify $a$ with gravitational acceleration, g,

$$
T=\frac{G M \hbar}{R^{2} k c} .
$$

One would therefore conclude that the absolute temperature is proportional to the mass were it not for the fact that $R$ is now the Schwarzschild radius,

$$
R=\frac{2 G M}{c^{2}} \text {. }
$$

Introducing (21) into (20) gives

$$
T=\frac{c^{3} \hbar}{4 k G M} \text {. }
$$

In contrast to $(20)$, where the temperature appears to be proportional to the mass $M,(22)$ now has it inversely proportional to it!

Confusing the rest energy, $M c^{2}$, with the internal energy and using the second law,

$$
\frac{\mathrm{d} S}{\mathrm{~d} E}=\frac{1}{T}=\frac{4 k G E}{c \hbar},
$$

one obtains an absolute entropy [10],

$$
S=2 k \frac{E^{2}}{E_{\mathrm{Pl}}},
$$

upon integrating and setting the arbitrary constant of integration equal to zero, where $E_{\mathrm{Pl}}=\sqrt{\hbar c^{5} / G}$ is the

${ }^{1}$ The integral, $\int_{0}^{\infty} \frac{x^{\nu-1} \mathrm{~d} x}{\mathrm{e}^{\mu x}-1}=\frac{1}{\mu^{v}} \Gamma(v) \zeta(v)$, exists for $\Re \mu>0$ and $\Re v>1$ [7]. 
Planck energy.

Since the entropy is the square of the energy, or mass, and the mass is proportional to the Schwarzschild radius, the entropy is proportional to the area, $A$, of the event horizon. Prior to making the identifications, and in units where all the universal constants are equal to one, the infinitesimal Euler relation would read [11]

$$
\mathrm{d} M=\frac{\kappa}{8 \pi} d A+\boldsymbol{\Omega} \cdot d \boldsymbol{J},
$$

for a neutral, rotating black hole at an angular velocity $\boldsymbol{\Omega}$, angular momentum $\boldsymbol{J}$. The surface gravity, which is subsequently set equal to the absolute temperature is $\kappa=1 / 4 M$.

We will now follow Page's [12] presentation of Hawking's calculation of black-hole emission for free fields, since it has a hindsight of thirty years. Hawking found the expected number of particles, $\bar{n}$, emitted in a wave mode of frequency, $\omega$, angular momentum, $\boldsymbol{j}$, as

$$
\bar{n}=\frac{\Gamma}{\mathrm{e}^{2 \pi \kappa^{-1}(\omega-\Omega \cdot j)}-1},
$$

where $\Gamma \leq 1$ is the absorption probability for an incoming wave.

For the entropy of radiation, Hawking took the von Neumann entropy for the thermal density matrix of each mode $^{2}$,

$$
\delta S_{\mathrm{rad}}=-\sum_{n} p_{n} \ln p_{n}=(\bar{n}+1) \ln (\bar{n}+1)-\bar{n} \ln \bar{n},
$$

since the probabilities are

$$
p_{n}=\frac{\bar{n}^{n}}{(\bar{n}+1)^{n+1}} .
$$

The expected loss in the black-hole entropy from the emission mode, due losses in energy, $\bar{n} \omega$, and angular momentum, $\bar{n} \boldsymbol{j}$, is

$$
\delta S_{\mathrm{bh}}=-\bar{n}\left[2 \pi \kappa^{-1}(\omega-\boldsymbol{\Omega} \cdot \boldsymbol{j})\right]=-\bar{n} \ln \left(\frac{\Gamma+\bar{n}}{\bar{n}}\right) .
$$

The second law is satisfied since the total change in entropy from radiation plus angular momentum loss due to emission is

$$
\begin{aligned}
\delta S & =\delta S_{\mathrm{rad}}+\delta S_{\mathrm{bh}} \\
& =\ln (\bar{n}+1)+\bar{n} \ln \left(1+\frac{1-\Gamma}{\Gamma+\bar{n}}\right) \geq \ln (\bar{n}+1) \geq 0,
\end{aligned}
$$

"with the extreme right inequality being saturated only if there is no emission, $\bar{n}=0$ " [12].

"Thus, the Hawking emission from a black-hole into

\footnotetext{
${ }^{2}$ Hawking makes allowance for fermions in all his expressions. However, fermions, or for that matter any particles other than photons, do not have a pure black-body spectrum, so the generalization and particle emission are illusory.
}

empty space obeys the second law of thermodynamics, and it actually produces entropy from all modes with nonzero emission. This is as one would expect, since the emission from a black-hole with $T_{\mathrm{bh}}>0$ into empty space with $T=0$ is an out-of-equilibrium process" [12].

Rather than using the second law of thermodynamics, the effective temperature is obtained from the Boltzmann factor,

$$
\frac{p_{1}}{p_{0}}=\frac{\bar{n}}{\bar{n}+1}=\mathrm{e}^{-\omega / T},
$$

which upon taking logarithms of both sides yields

$$
T=\omega / \ln \left(\frac{\bar{n}+1}{\bar{n}}\right) .
$$

For a Schwarzschild black-hole, or " $s$-waves of neutral particles in any Kerr-Newman geometry", the Planck factor becomes $1 /\left(\mathrm{e}^{2 \pi \omega / \kappa}-1\right)$, and "when $\Gamma=1$, so that the classical incoming wave is totally absorbed by the blackhole, then $T=T_{\mathrm{bh}}=\kappa / 2 \pi$, the Hawking temperature of the hole. But, otherwise, the effective temperature $T$ for the mode generically depends on the mode" [12].

Parenthetically, the form of the inverse temperature given by (30) is incompatible with the black-hole expression (23). This becomes apparent when we write the average energy as $\bar{E}=\bar{n} \omega$ in (30),

$$
\frac{\omega}{T}=\ln \left(1+\frac{\omega}{\bar{E}}\right) \approx \frac{\omega}{\bar{E}}
$$

for $\bar{n} \gg 1$, in the Rayleigh-Jeans limit. Expression (31) asserts that the absolute temperature is proportional to the average energy, and not inversely proportional to it as (23) claims. Moreover, if we attempted to apply the second law to (28) we would not come out with (30) because (28), or its negative, is not an entropy, but only part of one. Even worse, (28) has nothing whatever to do with the Bekenstein expression (24) for the entropy of a black-hole. For if it did, (31) could be integrated to give that expression in the same way that (23) is integrated to give (24).

By the conservation of energy and angular momentum, the rate that the black-hole loses them is equal to the rate that radiation gains them. No so for the total entropy, (29), which gains more than it loses. The rate of change of black-hole entropy is given as

$$
\frac{\mathrm{d} S_{\mathrm{bh}}}{\mathrm{d} t}=\frac{1}{2 \pi} \int_{0}^{\infty}\left[2 \pi \kappa^{-1}(\omega-\boldsymbol{\Omega} \cdot \boldsymbol{j})\right] \bar{n} \mathrm{~d} \omega .
$$

Let us begin our critique with the first law, (25), which black-hole thermodynamicists would write as

$$
\mathrm{d} E=T \mathrm{~d} S+\boldsymbol{\Omega} \cdot \mathrm{d} \boldsymbol{J} .
$$

Distinction must be made between the energy, $E$, in a fixed coordinate system, and the energy, 


$$
E^{\prime}=E-\boldsymbol{\Omega} \cdot \boldsymbol{J},
$$

in a coordinate system rotating with the body [13], in this case a Kerr black-hole. Since the adiabatic definition of angular momentum is

$$
\left(\frac{\partial E^{\prime}}{\partial \boldsymbol{\Omega}}\right)_{S}=-\boldsymbol{J}
$$

it follows from (35) that the differential for the energy in a rotating coordinate system is

$$
d E^{\prime}=T d S-\boldsymbol{J} \cdot d \boldsymbol{\Omega} .
$$

Differentiating (34) and introducing it into (36) gives (33).

Likewise, the differential for the Helmholtz free energy,

$$
F^{\prime}=E^{\prime}-T S,
$$

in a rotating coordinate system is

$$
d F^{\prime}=-S d T-\boldsymbol{J} \cdot d \Omega,
$$

while that in a fixed coordinate system is

$$
d F=-S d T+\boldsymbol{\Omega} \cdot d \boldsymbol{J} .
$$

Kirchhoff made use of the general thermodynamic law that radiation cannot upset a state of thermal equilibrium. For black-body radiation, Kirchhoff showed that, at thermal equilibrium and in each frequency interval, there is an equality between emitted, $e_{\omega}$, and absorbed, $a_{\omega} K_{\omega}$, radiant energy, where $a_{\omega}$ is the fraction of absorbed energy in the frequency interval, $\omega$ and $\omega+d \omega$, and $K_{\omega} d \omega$ is the intensity of radiation in the same interval. This is expressed by Kirrchhoff's radiation law [1, p. 64]:

$$
e_{\omega}=a_{\omega} K_{\omega} .
$$

Hawking calculates the left-hand side of (40) and claims that this energy per unit frequency interval is dumped into empty space with zero temperature, constituting an out-of-equilibrium process. This is tantamount to calculating a reaction in the forward direction and forgetting to equate it with the rate of the reverse reaction in the law of mass action which secures chemical equilibrium. Likewise, at thermal equilibrium, the total emissive power must be equal to the total absorptive power. Kirchhoff was able to extend this equality to each and every frequency interval by considering cavities made of different materials. Moreover, Hawking considers a blackhole as a perfect black-body by setting $a_{\omega}=\Gamma=1$, but fails to take into account the form of the emissive power $K_{\omega}$,

$$
u_{\omega}=\frac{4 \pi}{c} K_{\omega}=\omega^{3} \phi(\omega / T),
$$

dictated by Wien's displacement law, where $u_{\omega}$ is the energy density, $K_{\omega}$ is the intensity in the frequency range from $\omega$ to $\omega+d \omega$, and $\phi$ is an unkown function of the single argument, $\omega / T$. It is precisely the pre-factor that is missing in (26) that is required in order to obtain Stefan's law upon integrating over all frequencies. The cavity may even have perfectly reflecting walls, $a_{\omega}=\Gamma=0$ provided it contains a speck of charcoal dust that, through absorption and emission, will allow an arbitrary distribution of energy to approach the equilibrium distribution, $u_{\omega}$. Therefore, Hawking is not calculating the emission of energy and angular momentum, but, rather, the equilibrium distribution of the average number of photons, provided the number of oscillators $m_{\omega}$ in the frequency interval from $\omega$ to $\omega+d \omega$ is inserted into the numerator of (26).

The expression used by Hawking to calculate the entropy of black-body radiation, (27), in the frequency interval from $\omega$ to $\omega+d \omega$ is the classical ShannonGibbs entropy that is compatible with Boltzmann statistics, and not the negative binomial distribution of blackbody radiation. At most, (27) accounts for the Wien limit. The entropy of black-body radiation in the interval from $\omega$ to $\omega+d \omega$ is not (27), but [1, p. 77 eqn (2.24)]

$$
S_{\mathrm{rad}}(\bar{n})=\bar{n} \ln \left(\frac{\bar{n}+m_{\omega}}{\bar{n}}\right)+m_{\omega} \ln \left(\frac{\bar{n}+m_{\omega}}{m_{\omega}}\right) .
$$

Just as $m_{\omega}=8 \pi \omega^{2} / c^{3}$ is the number of Planck oscillators in the frequency interval from $\omega$ to $\omega+d \omega$, so (42) is the entropy density in the same range. It is also not the change in entropy. This makes (32) blatantly wrong since an integration over all frequencies does not give the time rate of change of the black-hole entropy!

In view of the definition of the energy in a rotating coordinate system, (34), the definition of the change in the black-hole entropy, (28), is also wrong for it would require

$$
\delta S_{\text {bh }}=-2 \pi \kappa^{-1} \bar{E}^{\prime},
$$

where $\bar{E}^{\prime}=\bar{n}(\omega-\boldsymbol{\Omega} \cdot \boldsymbol{j})$, is just the average energy in the coordinate system rotating with the Kerr black-hole. The expression of the black-hole entropy (28) was thought to be justified by the fact that it gave back the first law. But, (43) shows that it gives only part of that law. Missing is the second expression in (42) which is the negative of the ratio of the average free energy to temperature, in the frequency interval $\mathrm{d} \omega$, [cf. eqn (37)], in the rotating coordinate system, viz.,

$$
\bar{F}^{\prime}=m_{\omega} T \ln \left(1-\mathrm{e}^{-\omega^{\prime} / T}\right),
$$

where $\omega^{\prime}=\omega-\boldsymbol{\Omega} \cdot \boldsymbol{j}$. Expression can easily be verified by calculating its derivative with respect to the temperature,

$$
\frac{\partial \bar{F}^{\prime}}{\partial T}=\frac{-\bar{n} \omega^{\prime}+\bar{F}^{\prime}}{T}=\frac{-\bar{E}^{\prime}+\bar{F}^{\prime}}{T}=-S_{\mathrm{rad}},
$$


which is (37).

Furthermore, the temperature, (30), can be obtained directly from the second law which is [1, p. 137]

$$
\frac{\mathrm{d} S_{\mathrm{rad}}}{\mathrm{d} \bar{n}}=\frac{\partial S_{\mathrm{rad}}}{\partial \bar{n}}+\frac{\partial S_{\mathrm{rad}}}{\partial \bar{E}} \frac{\mathrm{d} \bar{E}}{\mathrm{~d} \bar{n}}=\frac{\omega}{T}=\ln \left(\frac{\bar{n}+m_{\omega}}{\bar{n}}\right),
$$

since black-body radiation has zero chemical potential. Whereas the putative expression of a black-hole (28) is not related to the emissive power of a black-body, the entropy density (42) is. The appeal to Boltzmann statistics is an artifice since that statistics does not, in general, apply to black-body radiation as Planck realized. Furthermore, the black-hole entropy, (28), will not give the black-hole (body) temperature, (30), only (42) will when the degeneracy factor is properly accounted for.

\section{Are There Bounds on Entropy?}

Faced with the enormous numerical value of the entropy (24), Bekenstein [14] attempted to show that (24) was an upper limit for all entropies. For according to him, "There is no gap in magnitude between black-hole entropy and ordinary entropy. This comes about because of the existence of a hitherto unnoticed upper bound to the entropyto-energy ratio of non-black-hole systems of given effective radius $R$ ",

$$
\frac{S}{E}<k \frac{R}{\hbar c} .
$$

"For systems of negligible self-gravity, inequality (47) keeps $S$ from growing faster than $E$, a well-known property of ordinary bodies which is responsible for the seeming gap between this entropy and black-hole entropy (which grows as $E^{2}$ ). However, as one compresses a body to its gravitational radius, $R$ becomes of order $2 E$, and $S$ can begin growing as $E^{2}$ thus 'catching up' with black-hole entropy". No matter what the effect of gravitational compression may be, it cannot change a concave function into a convex one.

"Evidently, systems composed of nonrelativistic particles are not very interesting from the point of view of the bound" [14]. Therefore, the characteristic radius can be taken as the relativistic thermal wavelength, $R=\hbar c / k T$, so that (47) is actually

$$
\frac{S}{E}<\frac{\mathrm{d} S}{\mathrm{~d} E},
$$

on the strength of the second law. Then, the whole question boils down to is whether $S$ is an increasing or decreasing function of $E$. Because of the universality of the entropy it cannot be both. According to a theorem on twice differentiable functions [15], $S=E^{k}$ will be convex in $(0, \infty)$ if $k<0$ or $k>1$, and concave if $0<k<1$. Hence,

$$
\frac{S}{E}=E^{k-1}>\frac{\mathrm{d} S}{\mathrm{~d} E}=k E^{k-1},
$$

precisely because $k<1$.

The defining property of the entropy is its concavity [ 1 , 16]. We consider the entropy as a sole function of the energy, and use primes to denote derivatives. If

$$
E S^{\prime}-S \leq 0 \text { and } \frac{\mathrm{d}}{\mathrm{d} E}\left(E S^{\prime}-S\right)=E S^{\prime \prime}<0,
$$

then $S / E$ decreases. If $E_{1}$ and $E_{2}$ are any two values of the energy, the concavity of the entropy requires:

$$
S\left(\lambda E_{1}+(1-\lambda) E_{2}\right) \geq \lambda S\left(E_{1}\right)+(1-\lambda) S\left(E_{2}\right),
$$

for $0<\lambda \leq 1$. In particular, the slope of a straight line from the origin to any point on the entropy curve, say $E$, cannot be inferior to the tangent of the curve at that point. We thus set $E_{1}=E$ and $S\left(E_{2}\right)=0$ in (51), and obtain

$$
\frac{S(\lambda E)}{\lambda E} \geq \frac{S(E)}{E},
$$

where we have divided both sides of the inequality by $\lambda E$. Since $E>\lambda E,(52)$ shows that that the ratio $S / E$ decreases.

As a prime example of where to apply his inequality (47), Bekenstein considers black-body radiation for which

$$
\frac{S}{E}=\frac{4}{3}\left(\frac{V}{E}\right)^{\frac{1}{4}}=\frac{4}{3 T} \text {. }
$$

He then realizes that the ratio of entropy-to-energy "can be made as large as we please simply by lowering $T$ sufficiently. But in fact the thermodynamic description of radiation on which $[(53)]$ is based breaks down when $T$ is no longer large compared to the reciprocal of the characteristic size of the system (typical wavelength not small compared to cavity size). Boundary effects make themselves felt. These can be expected to arrest the growth of $S / E$ as $T$ is lowered further". On the contrary, black-body radiation makes no such demands: the integral is over all wavelengths from 0 to $\infty$ !

The first expression in (50) is the Massieu transform of the entropy with respect to energy, which is the product of the Helmholtz free energy, $F$, and the inverse temperatue $\beta$,

$$
E S^{\prime}-S=\beta F=-\ln \mathcal{Z}(\beta) \leq 0,
$$

where $\mathcal{Z}$ is the partition function. For an ideal gas $\mathcal{Z}$ is a monotonic decreasing function of $\beta$, whereas for black-body radiation $\ln \mathcal{Z}$ is the same decreasing function of $\beta$,

$$
\ln \mathcal{Z}(\beta)=\frac{1}{3} \sigma \beta^{-3} V,
$$

where $\sigma$ is the Stefan-Boltzmann constant, and $V$ is 
the volume of the cavity containing the radiation. According to Bekenstein, "the wanted distribution is the canonical one whose inverse temperature is just the peak value of $S / E$ for the system". Since

$$
S=\beta E+\ln \mathcal{Z},
$$

(which is a rearrangement of (54)) an equality in (48), would be given by the condition that

$$
\ln \mathcal{Z}\left(\beta_{0}\right)=0 \text {, }
$$

since $\beta_{0}=(S / E)_{\max }$.

Unfortunately, there is no finite value of $\beta_{0}$ that would satisfy (57) for black-body radiation where the logarithm of the partition function is given by (55). If such a value could be found, (54) would accomodate both concave entropies, for which $F<0$, and convex entropies, for which $F>0$. Since $\ln \mathcal{Z}$ is a monotonic decreasing function of $\beta$, the conclusion does not follow that "the problem is thus superfically simple; the maximal $S / E$ is just that $\beta$ for which the partition function is unity".

The second inequality in (50) ensures that the heat capacity at constant volume is positive, viz.,

$$
S^{\prime \prime}=-\left(T^{2} C_{V}\right)^{-1}<0 .
$$

Inequality (58) is violated by the Bekenstein entropy expression, (24), since

$$
S^{\prime \prime}=\frac{4 k}{E_{\mathrm{Pl}}^{2}}>0 .
$$

The heat capacity at constant pressure cannot be defined for black-body radiation because black-body radiation is a phase equilibrium insofar as the number of photons is not conserved [1,17]. The pressure, $p$, is a sole function of the temperature and obeys the CarnotClapeyron equation,

$$
\frac{\mathrm{d} p}{\mathrm{~d} T}=\frac{h}{T},
$$

where $h$ is the enthalpy density, or the latent heat of sublimation. In fact, the Carnot-Clapeyron relation (60) leads at once to Stefan's law of black-body radiation once the thermal equation of state, $p=\frac{1}{3} \varepsilon$, is introduced where $\varepsilon=E / V$, the energy density.

Hawking [9] claims that tiny black-holes will radiate away at a temperature (20) leading to complete evaporation in an explosion that would result in an intense burst of photons in the x-ray region. It is well-known that saturated vapor pressure depends only on the temperature, and not upon volume. Changing the volume of vapor pressure at constant temperature will result in evaporation or condensation so as to leave the vapor pressure constant. This is the physical content of (60). Evaporation would mean the disappearance of the photon phase leading to a rupture in the phase equilibrium. For the processes of evaporation or condensation to proceed irreversibly, the pressure of the two phases cannot be equal but differ by the capillary pressure. An increase in volume of cavity radiation can be thought of as new radiation being evaporated from the walls of the cavity $[1,17]$. It is precisely the job of the latent heat $h$ to maintain the walls at constant temperature. If a black-body would absorb more radiation than it emits, it would heat up and the radiation would no longer be (iso) thermal radiation.

\section{Does Inflation Violate Einstein's Equations?}

It has been said that inflation is an elegant way of explaining why the universe is so homogeneous on the Hubble scale [18]. In this section, we show the contrary.

The Robertson-Walker (RW) metric,

$$
d s^{2}=R^{2}(t)\left\{d \chi^{2}+\sin ^{2} \chi\left(d \theta^{2}+\sin ^{2} \theta d \phi^{2}\right)\right\},
$$

where $R(t)$, the expansion, or scale, factor, is said to describe a space that is both homogeneous and isotropic. The question is: Why is space homogeneous and isotropic? It is this question that inflationary cosmology hoped to answer. But before we get to that scenario, there are criticisms to be lodged against the RW metric, (61).

The RW metric (61) is a metric of constant curvature. Introducing $r=R \sin \chi \quad$ [19] in (61) gives

$$
d s^{2}=\left\{\frac{d r^{2}}{1-r^{2} / R^{2}}+r^{2}\left(d \theta^{2}+\sin ^{2} \theta d \phi^{2}\right)\right\},
$$

where the scalar curvature $1 / R^{2}$ can be chosen as +1 , -1 , or 0 for positive, negative, or zero spatial curvature, respectively. It is well-known that the transition from spherical to hyperbolic geometry is allow the radius to become imaginary, $R \rightarrow \mathrm{i} R$. This would transform the RW metric (61) into

$$
d s^{2}=R^{2}(t)\left\{d \chi^{2}+\sinh ^{2} \chi\left(d \theta^{2}+\sinh ^{2} \theta d \phi^{2}\right)\right\},
$$

but $r \neq R \sinh \chi$, as it is assumed in almost all texts on cosmology [19, eqn (111.12)].

The hyperbolic measure of distance is well-known to be given by $\chi=\tanh ^{-1} r / R$, so that when it is introduced into (63) it will give the well-known Beltrami metric [5, p. 494]

$$
\mathrm{d} s^{2}=\frac{\mathrm{d} r^{2}}{\left(1-r^{2} / R^{2}\right)^{2}}+\frac{r^{2}}{1-r^{2} / R^{2}}\left(\mathrm{~d} \theta^{2}+\sin ^{2} \theta \mathrm{d} \phi^{2}\right) .
$$

With the substitution $R \rightarrow \mathrm{i} R$, the transformation $r=$ $R \tan \chi$ would not give the RW metric, (61). The deviation from Euclidean geometry can be seen very clearly from 
(64) by considering $r=$ const, and $\theta=\pi / 2$. For then, the ratio of the periphery of a circle to the radius will not be $2 \pi$, but $^{3}$

$$
\frac{2 \pi}{\sqrt{1-r^{2} / R^{2}}}>2 \pi
$$

since $R \sinh \chi=r / \sqrt{1-r^{2} / R^{2}}$. In constrast, for elliptic space the ratio would be less than $2 \pi$,

$$
\frac{2 \pi}{\sqrt{1-r^{2} / R^{2}}}<2 \pi
$$

because $R \sin \chi=r / \sqrt{1+r^{2} / R^{2}}$. It is rather curious that since the metric (61) bears the name of Robertson that the only place where it is mentioned in his book, Relativity and Cosmology, is in the forward by Fowler. What Robinson refers to as the cosmological metric is the Beltrami metric (64) [3, p. 342, eqn (14.12)], and its elliptical counterpart, obtained as usual by $R \rightarrow \mathrm{i} R$.

The starting point for the inflationary scenario is the pair of Einstein equations that have been derived from the RW metric, (61), which are, quite remarkably, the same for the Beltrami metric, (64). The expressions for the energy density [3, p. 372],

$$
\kappa \varepsilon=3 \frac{\dot{R}^{2}+k}{R^{2}}-\Lambda,
$$

and pressure

$$
\kappa p=-\frac{2 R \ddot{R}+\dot{R}^{2}+k}{R^{2}}+\Lambda,
$$

where $\kappa=8 \pi G / c^{2}$, are given in terms of the cosmic time derivatives of the expansion factor $R, k$, the constant space curvature, and $\Lambda$, the cosmological constant.

Between the energy density and pressure there is the thermal equation of state [20],

$$
\eta \varepsilon=p
$$

where normally $\eta \in\left[\frac{1}{3}, \frac{2}{3}\right]$, the limits being the ultrarelativistic and non-relativistic non-interacting particle limits, respectively.

From thermodynamics we have the infinitesimal Euler relation,

$$
d E=T d S-p d V,
$$

where $S$ is the entropy. Choosing $V, T$ as the independent variables, differentiating with respect to the volume, $V$, and using a Maxwell relation result in $[21,22]$

$$
\left(\frac{\partial E}{\partial V}\right)_{T}=T\left(\frac{\partial p}{\partial T}\right)_{V}-p
$$

${ }^{3}$ For a history of the uniformly rotating disc in relativity consult in $[5$, $\S 9.1]$.
Employing the thermal equation of state, (67), to eliminate the pressure gives linear partial differential equation of first-order,

$$
T\left(\frac{\partial E}{\partial T}\right)_{V}-\frac{V}{\eta}\left(\frac{\partial E}{\partial V}\right)_{T}=E
$$

Lagrange [23] reduced the method of solving the linear partial differential Equation (69) to one of solving the auxiliary set of ordinary differential equations,

$$
\frac{\mathrm{d} T}{T}=\frac{\mathrm{d} E}{E}=-\eta \frac{\mathrm{d} V}{V},
$$

whose solutions, the characteristic curves, fill the (T, $V$, $E)$-space. The vector,

$$
\boldsymbol{v}=(T,-V / \eta, E),
$$

will be tangent to the family of characteristic curves.

The general solution to (69) is

$$
\psi\left(c_{1}, c_{2}\right)=0,
$$

where $T V^{\eta}=c_{1}$ and $E V^{\eta}=c_{2}$, are the two independent solutions, and $\psi$ is an arbitrary function. The general solution can also be written as:

$$
E=V^{-\eta} \Psi\left(T V^{\eta}\right),
$$

where $\Psi$ is arbitrary function, which must be further conditioned by the thermodynamic stability conditions, e.g., $\Psi$ cannot be chosen as an inverse function for that would have energy decreasing with temperature. The solution (71) to (69) can be represented as a surface in $(T, V, E)$-space. Starting at any point on surface, (71), a curve can be traced out in the direction of the tangent vector (70). The surface, (71), can be thought of as being formed by the family of such characteristic curves.

Returning to (68), we can write it as:

$$
T d S=d E+p d V=d E+\frac{\eta E}{V} d V,
$$

with the aid of (67). It is readily seen that $V^{\eta}$ is an integrating factor for (72) since

$$
V^{\eta} T d S=d\left(E V^{\eta}\right)
$$

This allows us to express the entropy as

$$
S=\Phi\left(T V^{\eta}\right)
$$

where $\Phi$ is an arbitrary function, again with the caveat that it must not violate thermodynamic stability criteria.

The Einstein equations, (65) and (66), can be combined into [3, p. 373]

$$
\frac{\mathrm{d}}{\mathrm{d} t} \varepsilon R^{3}=-p \frac{\mathrm{d}}{\mathrm{d} t} R^{3},
$$

which, on the strength of (68), is the condition of adia- 
baticity. The fundamental error [24] in both the old [25], and new [26,27], inflationary cosmologies is to consider (75) as a separate condition distinct from

$$
\frac{\mathrm{d}}{\mathrm{d} t} s R^{3}=0
$$

where $S$ is the entropy density. As can be seen from (68), (76) is not independent of (75).

So given any expression for the energy density $\varepsilon$, the expression for the pressure $p$ can be found from (75), and Einstein's expressions for the energy density and pressure are consistent with (75). But, there is a new element now, the thermodynamic relation (71), which is independent of time derivatives. Although Einstein's equations express the energy density and pressure in terms of the time derivatives of the scale factor, they must necessarily be compatible with (71) if they are to have any thermodynamic relevance whatever.

Since Einstein's theory demands an adiabatic universe, it follows from (74) that

$$
T V^{\eta}=T R^{3 \eta}=\text { const. }
$$

Imposing this on (71) results in

$$
\varepsilon=\frac{E}{V}=\frac{C}{R^{3(1+\eta)}},
$$

where $C$ is a constant. Such a system is said to be repulsive $[21,22]$, because will not condense:

$$
\left(\frac{\partial E}{\partial V}\right)_{T}<0 .
$$

Expression (78) is exactly the solution to the energy conservation equation,

$$
\dot{\varepsilon}=-3 \frac{\dot{R}}{R}(\varepsilon+p),
$$

which is obtained when the energy-momentum tensor,

$$
T_{i}^{k}=(\varepsilon+p) u_{i} u^{k}+p \delta_{i}^{k},
$$

${ }^{4}$ However, when (81) is introduced into (82) there results,

$$
\frac{\partial T_{i}^{k}}{\partial x^{k}}=u_{i} \frac{\partial h u^{k}}{\partial x^{k}}+h u^{k} \frac{\partial u_{i}}{\partial x^{k}}+\frac{\partial p}{\partial x^{k}}=0,
$$

where $h=\varepsilon+p$ is heat content, or the enthalpy, density. It is unclear how by multiplying a zero-vector like (83) by the 4-velocity $u^{i}$ that it can it be projected on the direction of the 4-velocity [28]. In any event, since the 4-velocities satisfy $u^{i} u_{i}=-1$ so that $u_{i} \partial u^{i} / \partial x^{k}=0$, the energy balance condition,

$$
u^{i} \frac{\partial T_{i}^{k}}{\partial x^{k}}=-\frac{\partial h u^{k}}{\partial x^{k}}+u^{k} \frac{\partial p}{\partial x^{k}}=0
$$

is correct. Yet, the two conditions, (83) and (84) are not one and the same, for when (84) is introduced into (83) there results

$$
u_{i} u^{k} \frac{\partial p}{\partial x^{k}}+\frac{\partial p}{\partial x^{i}}+h u^{k} \frac{\partial u_{i}}{\partial x^{k}}=0
$$

is substituted into the Bianchi identity,

$$
\frac{\partial}{\partial x^{k}} T_{i}^{k}=0 .
$$

Thus, the Bianchi identities are consistent with an adiabatic universe ${ }^{4}$.

Introducing (78) into (65) gives

$$
3 R^{3 \eta+1}\left(\dot{R}^{2}+k\right)-\Lambda R^{3(\eta+1)}=\text { const. }
$$

Since the expansion factor depends on time, we must necessarily set $k=\Lambda=0$, because (85) must be homogeneous of order zero in time. Then $(85)$ reduces to

$$
R^{3 \eta+1} \dot{R}^{2}=\text { const. }
$$

The perfect cosmological principle asserts that the universe looks the same to any observer in motion [3, p. 347]. It requires $R=$ const ., or $\dot{R} / R=$ const, with $k=0$. The latter is the de Sitter model in which an empty universe expands exponentially. According to (65) and (66), the energy density is a constant, and the pressure is $p=-\varepsilon=$ const . This equation of state is said to characterize the vacuum [20]. The same conclusion can be reached by considering solely the cosmological constant in those expressions, and that is precisely what de Sitter did [29]. The problem lies with Einstein's equations for they cannot accomodate an energy density or pressure in a stationary universe.

Alternatively, in the standard model, the Hubble parameter, $H=\dot{R} / R$ falls off as $t^{-1}$, which means that $R=A \sqrt{t}$, where $A$ is a constant. This requires that we take $\eta=\frac{1}{3}$ in (86) which then reduces to $R \dot{R}=$ const, which not the virial theorem. Moreover, although the expansion factor increases in time, it will be decelerating because $\ddot{R}=-A / 4 \sqrt[3]{t}$. In short, the condition (86) is entirely ad hoc since it does not correspond to any known theorem, apart from $\eta=0$, which is the virial theorem.

The expression for the energy density (65) can be made to look like a generalization of the relativistic virial theorem, and would seem to satisfy (86) for $\eta=0$. For upon introducing $\varepsilon=M c^{2} /\left(4 \pi R^{3} / 3\right)$ into (65), there would result

$$
\frac{1}{2} \dot{R}^{2}=\frac{G M}{R},
$$

which is the relativistic virial ${ }^{5}$. The second of Einstein's equations is given by (66), again with $k=\Lambda=0$, but this time with $p=0$, viz.,

$$
\ddot{R}=-\frac{G M}{R^{2}},
$$

which is Newton's law. Therefore, $p \neq 0$ will cause

\footnotetext{
${ }^{5}$ The nonrelativistic virial would see the constant $\kappa$ halved.
} 
deviations from Newton's law.

Differentiation of (65) with respect to cosmic time $t$, and use of the adiabatic condition in the form (80) give

$$
\ddot{R}=-\frac{\kappa}{6}(\varepsilon+3 p) R=-\frac{\kappa}{6}(1+3 \eta) \varepsilon R .
$$

The equation of motion (89) can be written as

$$
\ddot{R}=-\frac{G M}{R^{2}}(1+3 \eta),
$$

so that $\eta \neq 0$ appears as a correction term to Newton's law. In fact,

$$
3 \eta=\frac{3 p}{\varepsilon}=\frac{2 G M}{R c^{2}},
$$

is equation of state for degenerate matter. The ratio of the pressure to rest energy density is the same order as the ratio of the Schwarzschild radius to the radius of a star, which is of the same order of magnitude as the red shift, the mass defect, and the deflection of light around white dwarfs [30]. For normal relativistic stars, $\eta$ is of the order $10^{-6}$, while for white dwarfs it is $10^{-4}$, reaching unity in the case of a black-hole. Therefore, (67) can hardly be considered a thermal equation of state where $\eta \in\left[\frac{1}{3}, \frac{2}{3}\right]$.

This is because (91) has nothing to do with the thermal equation of state (67), although many would contest this fact [20]. There is a big distinction between the rest energy density, $n m c^{2}$, where $n$ is the number density, and the internal energy $\varepsilon$. The distinction is blurred by writing the energy density and pressure as $[19$, p. 87$]$

$$
\varepsilon=n m \frac{c^{2}}{\sqrt{1-v^{2} / c^{2}}} \text { and } p=\frac{n m}{3} \frac{v^{2}}{\sqrt{1-v^{2} / c^{2}}},
$$

where the bar means an average over all particles. Then, in the non-relativistic limit, where terms are retained to order $v^{2} / c^{2}$, the thermal equation of state, (67), is reproduced with $\eta=\frac{2}{3}$. An average over all particles is confused with an average over a distribution of velocities, and the rest energy density has been omitted. As the above orders of magnitude show, the rest energy density is by far greater than the internal energy. In fact, the equation of state of (91) is [16, p. 41]

$$
\frac{p}{n m c^{2}}=\frac{2 G M}{c^{2} R}=\left(\frac{\lambda_{C}}{\lambda_{T}}\right)^{\gamma}
$$

where $\lambda_{C}=\hbar / m c$ is the Compton wavelength, and $\lambda_{T}$ is the thermal wavelength. In the non-relativistic limit $\gamma=2$ and $\lambda_{T}=\sqrt{\hbar / m k T}$, while, in the ultra-relativistic limit, $\gamma=1$ and $\lambda_{T}=\hbar c / k T$. In both cases, (93) be- comes an expression for thermal-gravitational equilibrium.

Finally, using (65) to eliminate the energy density gives

$$
\frac{R \ddot{R}}{\dot{R}^{2}}=-\frac{1}{2} \kappa(1+3 \eta) .
$$

The condition for acceleration, therefore, is [18, p. 395]

$$
1+3 \eta<0 \text {. }
$$

Under this condition, (86) shows that the kinetic energy will increase with the radius instead of decreasing with it as the virial theorem dictates. Moreover, Le Châtelier's principle is violated because (89) implies that due to a decrease in $R(t)$, the cosmic fluid will undergo compression, leading to an increase in the pressure which, in turn, aids the compression [3].

Because of the adiabatic condition, (77), there will be no type of phase transition envisioned by the inflationary cosmology. If we impose on (71) that the energy be extensive, we will get

$$
E=V T^{1+1 / \eta} .
$$

For the standard model, $\eta=\frac{1}{3}$, so that this model would identify (95) with Stefan's law of black-body radiation. Such a system is attractive and show signs of wanting to condense $[21,22]$ :

$$
\left(\frac{\partial E}{\partial V}\right)_{T}>0
$$

as opposed to our adiabatic system which will not condense because of (79). Black-body radiation needs a heat source to keep the walls of the cavity at a constant temperature. So it cannot occur in an adiabatic universe, unless part of that universe can be singled out as being in thermal equilibrium with the rest of the universe. The same distinction is made between the canonical (isothermal) ensemble which is embedded in a larger microcanonical (adiabatic) ensemble. This could explain CMBR, which would then not be a relic of a big bang.

Likewise, we require $p$ to be an intensive variable. Using (67) to evaluate (71) we obtain

$$
p=\frac{\left(T V^{\eta}\right)^{(1+\eta) / \eta}}{V^{1+\eta}}=T^{1+1 / \eta} .
$$

Differentiating (97) with respect to $T$ we get

$$
\frac{\mathrm{d} p}{\mathrm{~d} T}=\frac{(1+1 / \eta) p}{T}=\frac{h}{T},
$$

where $h$ is the enthalpy density, or the latent heat. The latent heat can be used to create a structural change in the system at the temperature $T$; if the volume is varied at constant temperature the liquid will either condense or 
evaporate so that the pressure will remain constant at that temperature. Latent heat is required, but it cannot be used to re-heat the system to just below the critical temperature after the phase transition ended [25] because the temperature remains constant. In short, latent heat cannot heat, so that inflationary cosmology must look for some other source to reheat the universe. Moreover, entropy "can[not] be generated in any phase transition" [25].

The Carnot-Clapeyron equation, (98), describes a phase equilibrium, and as such it violates the adiabatic condition that (74) be constant. It was first derived by Lord Rayleigh in an attempt to generalize Stefan's law to $1 / \eta$ dimensions $[1, \S 6.2]$. Thus, the phase transitions which the early universe was thought to have undergone could not have happened.

Be that as it may, the inflationary scenario confuses a first-order phase transition, in which there is latent heat, with a symmetry-breaking phase transition in which there is none. In the latter type of transition, discontinuities occur in the derivatives of thermodynamic potentials like the specific heat. New inflation [26,27] employs a scalar field, $\phi$, which was at one time displaced from the minimum of its potential, $\phi=0$ (the "false vacuum"), to a new symmetry-breaking minimum $\phi \neq 0 \quad[20, \S 8.2]$. The two states have a different symmetry. This is not a phase transition of the first kind where two different states are in equilibrium; a symmetry change occurs at the critical point so it is possible to say in which of the two phases the system belongs at any point of the transition.

The total energy consists of black-body radiation and an energy (zero-point) associated with the scalar field,

$$
\varepsilon=4 \sigma T^{4}+\varepsilon_{\phi}
$$

The free energy, $F=E-T S$, will have the same form as (71), namely,

$$
F=V^{-\eta} \Xi\left(T V^{\eta}\right),
$$

where $\Xi$ is an arbitrary function. Like (71), (100) expresses a dichotomy: Either $\Xi=$ const ., and the system has a zero-point energy and will not condense, or it is not constant and the system will condense as $T \rightarrow 0$. If the system has a zero-point energy,

$$
\left(\frac{\partial p}{\partial T}\right)_{V}=0
$$

showing that it will not condense. The entropy [21],

$$
S=-\left(\frac{\partial F}{\partial T}\right)_{V}=\int\left(\frac{\partial p}{\partial T}\right)_{V} \mathrm{~d} V+C,
$$

will then be a constant, $C$, or zero, in accordance with the third law. Yet, the zero-point energy in (99) leads to a condensation even at absolute zero, for

$$
\left(\frac{\partial E}{\partial V}\right)_{T=0}=\varepsilon_{\phi}>0,
$$

since $E=\varepsilon V$. The conclusion to be drawn is that $a$ zero-point energy in (99) (repulsion) is incompatible with the simultaneous presence of black-body radiation (attraction).

\section{Is There Gravitational Aberration?}

It is known that the absence of gravitational aberration requires a diminution in the magnitude of gravitational acceleration, just as if the electric field is to point in the direction of the charge, and not in its past position [31]. This is contested by the fact that light propagation is not collinear with its gravitational force [32], and the delayed time of propagation of the gravitational force would cause a couple to form between celestial bodies that would destroy their otherwise stable orbits. The reason why the field points to the instantaneous position of the source is thought to be a consequence of an exact cancellation of, at most, linear terms in the velocities. What is changed is the magnitude of the field, not its direction.

In the case of the Schwarzschild metric, the acceleration will be directed to the instantaneous position of a moving source of mass $M$ and velocity $v$. Thus, no aberration occurs, but there will be a decrease in magnitude of the acceleration by an anount [31]

$$
\begin{aligned}
\ddot{R} & =-\frac{G M}{\left(1-\frac{G M}{2 c^{2} R}\right)\left(1+\frac{G M}{2 c^{2} R}\right)^{5} R^{2}} \\
& =-\frac{G M}{R^{2}}\left(1-\frac{2 G M}{c^{2} R}+\cdots\right),
\end{aligned}
$$

to linear order terms in the ratio, $v / c$. In comparison with (90), the last expression shows that the decrease in the magnitude of acceleration is caused by a negative pressure,

$$
3 \eta=-\frac{2 G M}{R c^{2}} .
$$

which is the equation of state, (91), with the sign reversed. Curiously, the same effect has been attributed to gravitational aberration which would increase the angular momentum of an orbiting body thereby causing an expansion of the universe as a whole [33].

\section{REFERENCES}

[1] B. H. Lavenda, "Statistical Physics: A Probabilistic Approach," Wiley-Interscience, New York, 1991, p. 75.

[2] M. Planck, "Physikalische Abhandlungen und Vorträge," Braunschweig, Friedr. Vieweg \& Sohn, 1958, p. 599.

[3] H. P. Robertson and T. W. Noonan, "Relativity and Cosmology,” W. B. Saunders, Philadelphia, 1968, p. 374. 
[4] L. S. Schulman, "Techniques and Applications of Path Integration,” Wiley-Interscience, New York, 1981, p. 229.

[5] B. H. Lavenda, "A New Perspective on Relativity: An Odyssey in Non-Euclidean Geometries," World Scientific, Singapore, 2011.

[6] P. M. Ashling and P. W. Milonni, American Journal of Physics, Vol. 72, 2004, pp. 1524-1529.

[7] I. S. Gradshteyn and I. M. Ryzhik, "Table of Integrals, Series, and Products," Academic Press, Orlando, 1980.

[8] W. G. Unruh, Physical Review, Vol. D14, 1976, pp. 870892.

[9] S. W. Hawking, Nature, Vol. 248, 1974, pp. 30-31.

[10] J. D. Bekenstein, Physical Review, Vol. D7, 1973, pp. 2333-2346.

[11] K. S. Thorne, R. H. Pope and D. A. Macdonald, "Black Holes: The Membrane Paradgim," Yale U. P., New Haven CT, 1986, p. 39.

[12] D. N. Page, "Hawking Radiation and Black-Hole Thermodynamics," 2004, arXiv:hep-th/0409024v3.

[13] L. D. Landau and E. M. Lifshitz, "Statistical Physics," 2nd Edition, Pergamon, Oxford, 1969, p. 72.

[14] J. D. Bekenstein, Physical Review, Vol. D23, 1981, pp. 287-298.

[15] G. H. Hardy, J. E. Littlewood and G. Pólya, "Inequalities," 2nd Edition, Cambridge U. P., Cambridge, 1952, p. 77.

[16] B. H. Lavenda, "Thermodynamics of Extremes," Albion, Chicester, 1995.

[17] J. T. Vanderslice, H. W. Schamp Jr. and E. A. Mason, "Thermodynamics," Prentice-Hall, Englewood Cliffs, 1966, p. 146.

[18] P. J. E. Peebles, "Principles of Physical Cosmology," Princeton U. P., Princeton, 1993, p. 392.

[19] L. D. Landau and E. M. Lifshitz, "The Classical Theory of Fields," 4th Edition, Pergamon, Oxford, 1975, p. 361.

[20] E. W. Kolb and M. S. Turner, "The Early Universe," Addison-Wesley, Reading, 1990, p. 49.

[21] H. Einbinder, Physical Review, Vol. 74, 1948, pp. 805808. doi:10.1103/PhysRev.74.805

[22] B. H. Lavenda, "A New Perspective on Thermodynamics," Springer, New York, 2009, § 6.2.

[23] P. Moon and D. E. Spencer, "Partial Differential Equations," D. C. Heath, Lexington, 1969, p. 87.

[24] B. H. Lavenda and J. Dunning-Davies, Foundations of Physics Letters. Vol. 5, 1992, pp. 191-196.

[25] A. H. Guth, Physical Review, Vol. D23, 1981, pp. 347356.

[26] A. D. Linde, Physics Letters, Vol. B108, 1982, pp. 389393.

[27] A. Albrecht and P. J. Steinhardt, Physical Review Letters, Vol. 48, 1982, pp. 1220-1223.

[28] L. D. Landau and E. M. Lifshitz, "Fluid Mechanics," Pergamon, Oxford, 1959, p. 501.

[29] W. de Sitter, "On the Relativity of Rotation," Proceedings of $K N A W$, Vol. 19, 1917, pp. 527-532.

[30] R. Sexl and H. Sexl, "White Dwarfs and Black Holes," Academic Press, New York, 1979, p. 55.

[31] M. Ibison, H. E. Puthoff and S. R. Little, "The Speed of Gravity Revisited," Preprint Physics/9910050.

[32] T. Van Flandern, Physics Letters, Vol. A250, 1998, pp. 111.

[33] M. Křížek and A. Šolcová, "How to Measure Gravitational Aberration," In W. Hartkopf, P. Harmanec and E. Guinan, Eds., Binary Stars as Critical Tools \& Tests in Contemporary Astrophysics: Proceedings of the 240th Symposium of the International Astronomical Union, Prague, 22-25 August 2006, Cambridge U. P., Cambridge, 2007, p. 389. 